\title{
Changes of Neuropathy Target Esterase Affect Phospholipid Homeostasis in SK-N-SH Cells
}

\author{
LI YU-YUAN ${ }^{1,2}$, WU YI-JUN ${ }^{1 *}$ \\ ${ }^{1}$ Laboratory of Molecular Toxicology, State Key Laboratory of Integrated Management of Pest Insects and Rodents, \\ Institute of Zoology, Chinese Academy of Sciences, 100101 Beijing, China \\ ${ }^{2}$ University of Chinese Academy of Sciences, 100049 Beijing, China
}

\begin{abstract}
Neuropathy target esterase (NTE), is a membrane protein located in the endoplasmic reticulum $(E R)$. NTE has the activity of phospholipase B and can catalyze the deacylation of phosphatidylcholine $(P C)$ and lysophosphatidylcholine (LPC) to glycerylcholine (GPC). It is phosphorylated and aged by organophosphorus compounds (OPs), that induce delayed neuropathy in humans and sensitive animals. Our previous study has reported that the disruption of ER phospholipid homeostasis caused by the NTE inhibition may contribute to the initiation of the organophosphate-induced delayed neurotoxicity (OPIDN), while it is unknown how the disturbed phospholipid homeostasis initiates OPIDN. It is difficult to change phospholipids in in vivo experiments. Therefore, an in vitro model is urgently needed to explain the role of phospholipid homeostasis disorders in OPIDN. In this study, we altered the expression of NTE in SK-N-SH cells and determined its phospholipid component by using HPLC-MS. Our results showed that the changes of NTE affected the levels of PC, sphingomyelin (SM), phosphatidylethanolamine (PE), phosphatidylserine (PS), lysophosphatidylserine (LPS), phosphatidylglycerol (PG), and phosphatidylinositol (PI). Our results were consistent with the in vivo results. Furthermore, our findings indicate that the $S K-N-S H$ cell model is a significantly useful method for the further research on how the changes of phospholipid homeostasis initiate the OPIDN, which is easier than the in vivo experiments in practice.
\end{abstract}

Keywords: NTE, Phospholipid homeostasis, SK-N-SH cells

\section{Introduction}

Neuropathy target esterase (NTE), formerly known as neurotoxic esterase [1] is the sixth member of the patatin like phospholipase family, also known as PNPLA6 [2]. Certain organophosphorus compounds (OPs) can cause a delayed neuropathy known as OP-induced delayed neuropathy (OPIDN). OPIDN requires to inhibit more than $70 \%$ of NTE activity. The inhibition and aging of NTE were the necessary events for OPIDN. NTE is composed of 1327 amino acid residues with a molecular weight of about $146 \mathrm{kDa}$. It can integrate with the membrane and have esterase activity. The amino acids in the active center are Asp960, Ser966 and Asp1086 [3].

NTE plays an important role in the nervous system and reproductive system. Its importance is mainly reflected in the following four aspects: first, NTE maintains the integrity and the stability of nerve axons during nerve development and differentiation [4-6]; Second, NTE plays an important role in catalyzing the degradation of lecithin and in maintaining the metabolic homeostasis of lecithin [7]. Third, NTE gene knockout can cause placental dysplasia, including trophoblastic dysplasia and angiogenesis disorders [8]. Fourth, NTE affects cell cycle and mammalian cell proliferation [9].

The physiological substrate of NTE in the body is lecithin, which can catalyze the deacylation of lecithin synthesized by CDP-choline pathway and maintain the metabolic balance of lecithin [10]. Tri$o$-cresyl phosphate (TOCP) is a neurotoxic OP. Recent studies found that by inhibiting NTE enzyme activity, TOCP not only affected phosphatidylcholine (PC), lysophosphatidylcholine (LPC) and glycerol phosphate choline (GPC) but also impacted the balance of other phospholipids, such as phosphatidylethanolamine (PE).

*email:wuyj@ioz.ac.cn 
Moreover, TOCP disturbed the endoplasmic reticulum phospholipids homeostasis by inhibiting NTE activity [11].

However, the changes in cytophospholipid composition caused by the changes in NTE are not clear. In this study, we used different NTE expression cells to determine the changes in phospholipid components in response to the changes in NTE.

\section{Materials and methods}

\subsection{Chemical reagents}

Dulbecco's modified Eagle's medium (DMEM) with high sugar was purchased from Sigma (St. Louis, MO, USA). Fetal bovine serum (FBS) was purchased from Chuanye Biosciences (Tianjin, China). Anti-NTE antibody (sc-30588) was purchased from Santa Cruz Biotechnology (Santa Cruz, CA, USA). Anti- $\beta$-actin (CW0096), anti-HA tag (CW0092), goat anti-mouse IgG (CW0102S), and goat antirabbit IgG (CW0103S) antibodies were purchased from ComWin Biotech (Beijing, China). Mipafox and phenyl valerate (PV) were synthesized by our lab as described by Johnson [12]. Paraoxon and ammonium formate were obtained from Sigma-Aldrich (St. Louis, MO, USA). The synthetic lipid standards were purchased from Avanti Polar Lipids (Alabaster, AL, USA) which had already been described in previous study [11]. High performance liquid chromatography (HPLC) grades methanol, $\mathrm{N}$-hexane, chloroform and isopropanol were obtained from CNW Technology (Shanghai, China). Transfection reagent VigoFect was purchased from Invitrogen Vigorous Biotechnology (Beijing, China).

\subsection{Cell culture and transfection}

Human neuroblastoma SK-N-SH cells (purchased from the Cell Center of Chinese Academy of Medical Sciences, Beijing, China) were cultured in DMEM medium. Cells were incubated at $37^{\circ} \mathrm{C}$ with $5 \% \mathrm{CO}_{2}$ in a carbon dioxide incubator. First, the control vector pCDNA3.1(+) and the expression constructs of pCNDA-NEST, and the pCDNA-aNTE were transfected into SK-N-SH cells at logarithmic growth stage with VigoFect; second, they were screened with $500 \mu \mathrm{g} / \mathrm{ml} \mathrm{G} 418$ for 3 weeks; then, the positive cell clones were detected by Western blotting and their NTE activity was observed. All plasmids were conserved in our lab.

\subsection{Neuropathy target esterase activity assay}

Cellular NTE activity was determined in homogenates of different NTE expression cells by the absorbance difference for PV hydrolysis between samples exposed to paraoxon and paraoxon plus mipafox, as previously described by Johnson [12].

\subsection{Western blotting analysis}

The cells were washed three times with cold PBS, harvested in the lysis buffer containing $50 \mathrm{mM}$ Tris- $\mathrm{HCl}$ with $\mathrm{pH} 7.5,150 \mathrm{mM} \mathrm{NaCl}, 1 \%$ Triton X-100, $1 \mathrm{mM}$ EDTA, $1 \mathrm{mM}$ PMSF and $1 \%$ protease inhibitors, and then sonicated on ice. Lysates were centrifuged at 3,000 $\times \mathrm{g}$ for $15 \mathrm{~min}$ at $4{ }^{\circ} \mathrm{C}$ and the loading buffer was added to the supernatants. The protein samples were boiled at $100^{\circ} \mathrm{C}$ for $10 \mathrm{~min}$ and electrophoresed on $10 \%$ SDS-polyacrylamide gels. Then the gels were transferred to polyvinylidene fluoride membranes (Millipore, Darmstadt, Germany). The membranes were blocked in 5\% non-fat milk $(\mathrm{w} / \mathrm{v})$ in Tris-buffered saline buffer containing $0.1 \%$ Tween 20 for $2 \mathrm{~h}$ at room temperature, incubated with the primary antibody at $4{ }^{\circ} \mathrm{C}$ overnight, then incubated with the horseradish peroxidase-conjugated secondary antibody for $2 \mathrm{~h}$ at room temperature. The primary antibodies anti-NTE (1:1000), anti-HA $(1: 1000)$, and anti- $\beta$-Actin (1:1000), and the secondary antibodies goat anti-mouse IgG (1:5000) and goat anti-rabbit IgG (1:5000) were used. Finally, the membranes were stained with standard ECL reagents and then photographs were taken by MicroChemi 4.2 (NDR, Israel). 


\subsection{Phospholipid extraction and phospholipid profile analysis}

Phospholipids were extracted from different NTE expression cells as described by Bligh and Dyer [13]. In brief, cells were collected and then homogenized for 40 times in $1.2 \mathrm{~mL}$ TE buffer by using a glass-homogenizer. One milliliter $(1 \mathrm{~mL})$ of the homogenate was placed in the test tube and $10 \mu \mathrm{L}$ homogenate was used for the protein concentration test. The following chemicals were added to the remaining homogenate by order: $3.75 \mathrm{~mL}$ of chloroform/methanol $(1 / 2, \mathrm{v} / \mathrm{v})$ mixture which contained an appropriate amount of internal standards [PC (14:1/14:1), LPE (17:1), PG (14:0/14:0), LPG (17:1), $\mathrm{PE}(14: 0 / 14: 0)], 1.25 \mathrm{~mL}$ chloroform and $1.25 \mathrm{~mL} \mathrm{KCl}(0.1 \mathrm{~mol} / \mathrm{L})$. After then, the remaining homogenate added with chemicals were centrifuged at 3,000 g for $10 \mathrm{~min}$. The upper aqueous phase and the lower organic phase were collected and the intermediate proteins were discarded. $1.88 \mathrm{ml}$ chloroform was added in the upper aqueous phase and then the organic phase in the lower layer was collected. The organic phases of the two collections were pooled together. The organic phase was slowly dried with nitrogen and was stored at $-20^{\circ} \mathrm{C}$ for later use. The phospholipid components were analyzed by HPLCMS as described previously [11, 14]. Partial least square discriminant analysis (PLS-DA) was performed with SIMCA-P 13.0.

\subsection{Statistical analysis}

The data were expressed as mean \pm standard deviation. Statistical analysis was performed using SPSS 19.0 software. Data were evaluated by one-way ANOVA. The Turkey test was used to compare the differences among the groups. $\mathrm{P}<0.05$ indicates the existence of significant differences.

\section{Results and discussions}

\subsection{Generation of different NTE expression cells}

In order to determine the role of NTE in phospholipid metabolism, we transfected SK-N-SH cells with pCDNA-aNTE and pCDNA-NEST, and screened the cells with either pCDNA-aNTE or pCDNA-NEST. The cell clones with either a stable expression of NTE antisense RNA or an over-expression of NEST were determined by the Western blotting analysis and the NTE activity assay. The positive cell clones were named as SH/dsRNA-NTE and SH/HA-NEST respectively. The expression of NTE was almost eliminated in the SH/dsRNA-NTE cells and a weak NTE band was detected by the Western blotting (Figure 1A). Similarly, the hNEST was virtually expressed in SH/HA-NEST and a HA-tag band was detected by the Western blotting (Figure 1B). In comparison with the SK-N-SH cells transfected with control vector pCDNA3.1(+), the NTE activity of SH/dsRNA-NTE cells was significantly decreased, while that of SH/HA-NEST cells was significantly increased. In specific, SH/dsRNA-NTE lost about $60 \%$ of NTE activity and the NTE activity of SH/HA-NEST increased $80 \%$ (Figure 1C). These results demonstrated that the modified expression of NTE was efficient and specific.

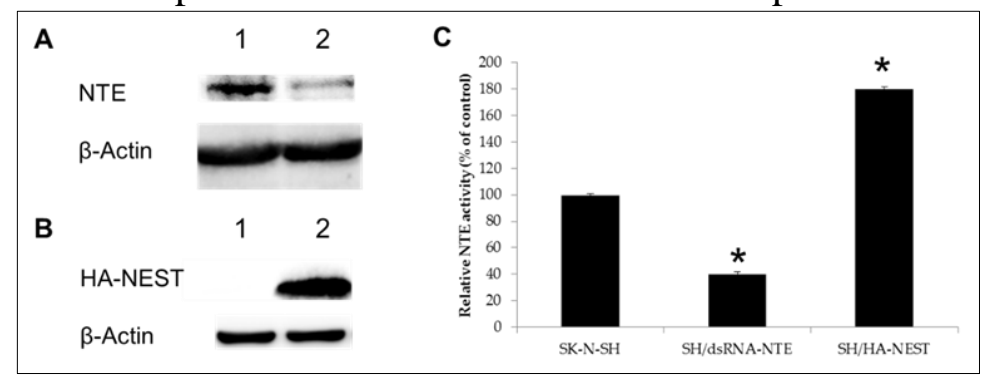

Figure 1. Determination of NTE expression and NTE activity in cells with different NTE expression levels. (A) WB was used to detect the expression of NTE. The first lane was the SK-N-SH cells and the second lane was SH/dsRNA-NTE cells. (B) WB was used to detect the NEST expression with HA tag. The first lane is the SK-N-SH cells and the second lane is SH/HA-NEST cells. (C) Determination of NTE activity. The data were expressed as a percentage of NTE activity in wild-type cells.

The NTE activity was significantly decreased in SH/dsRNA-NTE cells and increased in SH/HA-NEST cells $\left({ }^{*} \mathrm{p}<0.05, \mathrm{n}=3\right)$. 


\subsection{Comparative phospholipid omics}

To study the effect of the changes of NTE on phospholipid homeostasis, phospholipid omics analysis was performed in SK-N-SH, SH/dsRNA-NTE and SH/HA-NEST. 262 phospholipid species from 11 classes in total were identified, including PC, LPC, PE, sphingomyelin (SM), phosphatidylserine (PS), phosphatidylglycerol (PG), phosphatidylinositol (PI), lysophosphatidylethanolamine (LPE), lysophosphatidylserine (LPS), lysophosphatidylglycerol (LPG) and free fatty acid (FFA). The composition of phospholipids in SK-N-SH, SH/dsRNA-NTE and SH/HA-NEST were analyzed by PLS-DA. The PLSDA plot showed that the SK-N-SH was clearly separated from SH/dsRNA-NTE and SH/HA-NEST (Figure 2).

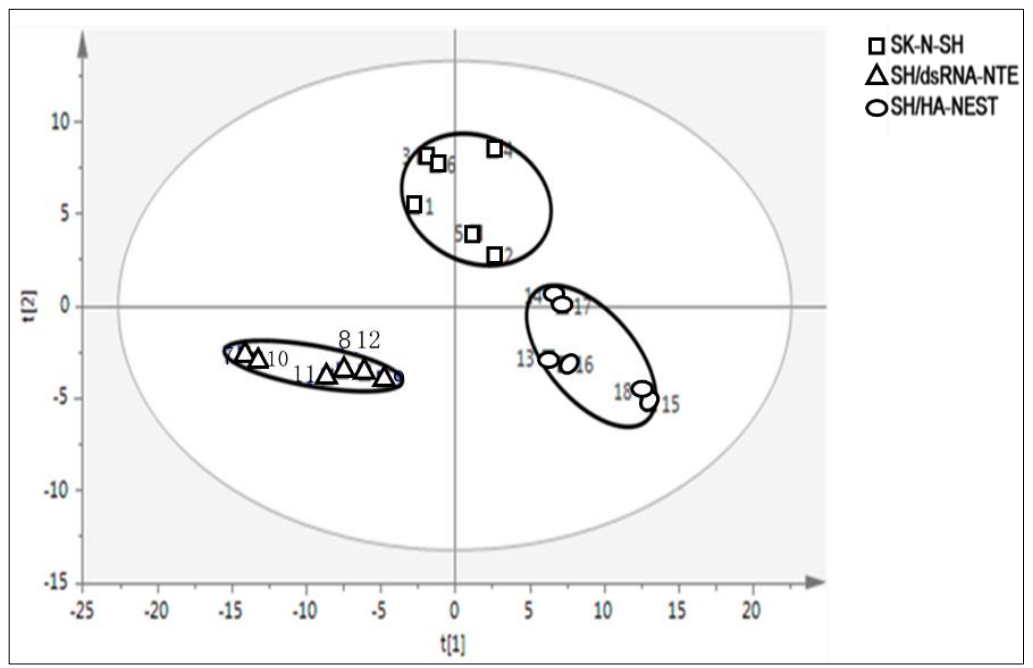

Figure 2. Phospholipid omics analysis of phospholipids from different NTE expression cells. PLS-DA score diagram of cellular phospholipid components.

Symbols: $\mathbf{\square}$, SK-N-SH; $\Delta$, SH/dsRNA-NTE; $\mathbf{O}$, SH/HA-NEST

Then the phospholipids that contributed the most to the separation of these three groups were determined by the variable importance plot (VIP) values. The VIP value is the weighted sum of the square of PLS weight, and reflects the importance of each variable to the whole model. When the VIP value is greater than 1.00 and the $\mathrm{P}$ value is less than 0.05 , there should be significant differences in 73 phospholipids among three groups. The specific results were shown in Table 1. In comparison with the SK-N-SH group, 50 out of 79 phospholipids in the SH/dsRNA-NTE group increased significantly, which belong to five classes: PI (5 phospholipids), PC (18 phospholipids), LPC (2 phospholipids), SM (11 phospholipids) and PS (5 phospholipids) (Figure 3A, white columns). While all of the other phospholipids in the SH/dsRNA-NTE group decreased significantly, most of which were PG and PE species (3 and 1, respectively) (Figure 3B, white columns). Meanwhile, the SH/HA-NEST group produced the opposite results to the SH/dsRNA-NTE group.

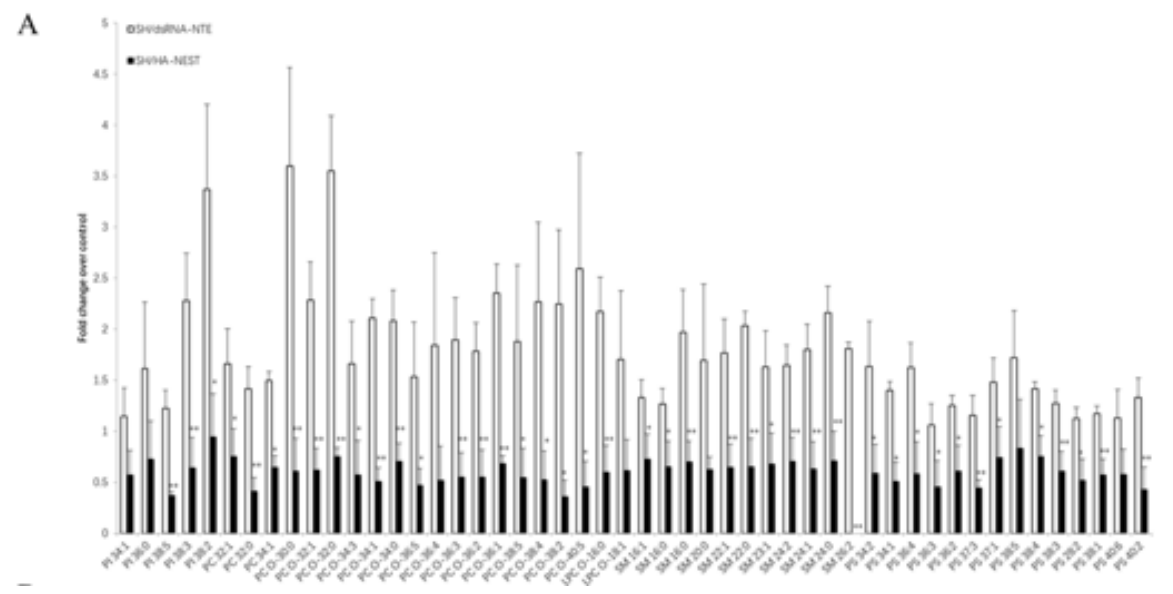




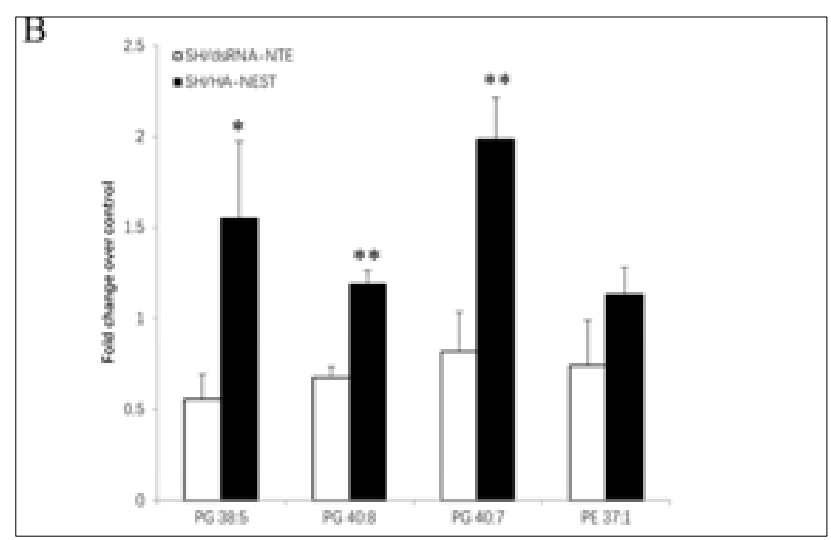

Figure 3. Phospholipid components that vary in cells with different NTE expressions. (A) Compared with the SK-N-SH, the phospholipid content in SH/dsRNA-NTE increased and the phospholipid content in SH/HA-NEST decreased. (B) Compared with the SK-N-SH, the phospholipid content in SH/dsRNA-NTE decreased and the phospholipid content in SH/HA-NEST increased. The white column represented the level of the phospholipid component in SH/dsRNA-NTE; The black column represented the level of the phospholipid component in SH/HA-NEST. Data were expressed as mean $\pm \operatorname{SEM}(n=6) .{ }^{*} \mathrm{P}<0.05$, and ${ }^{* *} \mathrm{P}<0.01$, compared to SH/dsRNA-NTE $(n=6)$

To further explain the effect of NTE changes on phospholipids, we compared the total phospholipids of various lipids in the three groups. In Table 2, the decreased expression of NTE induced significant increases of substrate PC content of NTE enzymes. The SH/HA-NEST group presented an opposite change to the SH/dsRNA-NTE group. At the same time, the decreased expression of NTE could induce the increase of SM, PI and PS, and reduce the content of PG, PE and LPS, while the over-expression of NTE caused the reverse change of the phospholipids mentioned above. In addition, the total amount of phospholipids in the three treatment groups remained unchanged.

Table 1. Phospholipids in different NTE expression cells (VIP $>1, \mathrm{P}<0.05$ ).

\begin{tabular}{cccccc}
\hline $\begin{array}{c}\text { Phospholipids } \\
\text { category }\end{array}$ & $\begin{array}{c}\text { Phospholipid } \\
\text { components }\end{array}$ & $\mathrm{m} / \mathrm{z}$ & $\begin{array}{c}\text { Retention time } \\
(\mathrm{min})\end{array}$ & VIP values & P values \\
\hline PC & $31: 0$ & 764.5447 & 48.966 & 1.77272 & 0.005 \\
& $32: 1$ & 776.5447 & 48.871 & 1.12657 & 0.002 \\
& $32: 0$ & 778.5604 & 48.860 & 1.04464 & 0.005 \\
& $33: 0$ & 792.5760 & 48.860 & 1.62634 & 0.010 \\
& $34: 1$ & 804.5760 & 48.682 & 1.04912 & 0.006 \\
& $38: 7$ & 848.5447 & 48.848 & 1.60859 & 0.030 \\
O-30:0 & 736.5498 & 48.824 & 1.25764 & 0.001 \\
& O-32:1 & 762.5655 & 48.765 & 1.20303 & 0 \\
& O-32:0 & 764.5811 & 48.646 & 1.31604 & 0 \\
& O-34:3 & 786.5655 & 48.717 & 1.07771 & 0.002 \\
& O-34:1 & 790.5968 & 48.480 & 1.14478 & 0.001 \\
& O-34:0 & 792.6124 & 48.468 & 1.25162 & 0 \\
& O-36:5 & 810.5655 & 48.575 & 1.02871 & 0.007 \\
& O-36:4 & 812.5811 & 48.457 & 1.03338 & 0.007 \\
& O-36:3 & 814.5968 & 48.386 & 1.14353 & 0 \\
& O-36:2 & 816.6124 & 48.338 & 1.10791 & 0.001 \\
& O-36:1 & 818.6281 & 48.314 & 1.22575 & 0.001 \\
& O-36:0 & 820.6437 & 48.314 & 1.29122 & 0.002 \\
& O-38:5 & 838.5968 & 48.314 & 1.02992 & 0.034 \\
& O-38:4 & 840.6124 & 48.303 & 1.1069 & 0.005 \\
& O-38:2 & 844.6437 & 48.184 & 1.10781 & 0.002 \\
& O-40:5 & 866.6281 & 48.196 & 1.09569 & 0.007 \\
\hline LPC & $16: 0$ & 540.3307 & 52.439 & 1.24123 & 0.011 \\
& $18: 1$ & 566.3463 & 52.261 & 1.6639 & 0.006 \\
& O-16:0 & 526.3514 & 52.771 & 1.21865 & 0 \\
& O-18:1 & 552.3671 & 52.617 & 1.05639 & 0.008 \\
\hline & $32: 0$ & 690.5079 & 16.267 & 1.52208 & 0.010 \\
\hline
\end{tabular}




\begin{tabular}{|c|c|c|c|c|c|}
\hline & $34: 1$ & 716.5236 & 15.876 & 1.00442 & 0.030 \\
\hline & $35: 3$ & 726.5089 & 22.644 & 1.48644 & 0.014 \\
\hline & $37: 1$ & 758.5705 & 14.941 & 1.04389 & 0.024 \\
\hline \multirow[t]{3}{*}{ LPE } & O-16:1 & 436.2834 & 27.515 & 1.12639 & 0.001 \\
\hline & O-18:2 & 462.2990 & 26.898 & 1.05684 & 0.004 \\
\hline & $17: 0$ & 466.2939 & 31.195 & 1.85752 & 0 \\
\hline \multirow[t]{5}{*}{ PG } & $33: 0$ & 735.5182 & 4.024 & 1.13194 & 0.007 \\
\hline & $38: 5$ & 795.5182 & 5.814 & 1.10072 & 0.001 \\
\hline & $40: 8$ & 817.5025 & 7.724 & 1.15977 & 0 \\
\hline & $40: 7$ & 819.5182 & 4.736 & 1.19006 & 0 \\
\hline & $42: 10$ & 841.5025 & 4.534 & 1.54667 & 0.001 \\
\hline \multirow[t]{17}{*}{ PS } & $34: 2$ & 758.4978 & 42.661 & 1.04915 & 0.006 \\
\hline & $34: 1$ & 760.5134 & 42.969 & 1.05105 & 0.004 \\
\hline & $34: 0$ & 762.5291 & 42.969 & 1.37101 & 0.006 \\
\hline & $36: 4$ & 782.4978 & 43.479 & 1.05602 & 0.005 \\
\hline & $36: 3$ & 784.5134 & 42.874 & 1.15815 & 0.011 \\
\hline & $36: 2$ & 786.5291 & 42.851 & 1.02023 & 0.011 \\
\hline & $37: 3$ & 798.5291 & 43.017 & 1.07329 & 0.010 \\
\hline & $37: 1$ & 802.5604 & 48.741 & 1.05215 & 0.007 \\
\hline & $38: 5$ & 808.5134 & 43.503 & 1.03179 & 0.024 \\
\hline & $38: 4$ & 810.5291 & 43.538 & 1.00225 & 0.019 \\
\hline & $38: 3$ & 812.5447 & 42.673 & 1.00565 & 0.013 \\
\hline & $38: 2$ & 814.5604 & 42.685 & 1.07489 & 0.013 \\
\hline & $38: 1$ & 816.5760 & 42.898 & 1.05872 & 0.009 \\
\hline & $40: 6$ & 834.5291 & 42.709 & 1.00432 & 0.041 \\
\hline & $40: 5$ & 836.5447 & 49.014 & 1.35925 & 0 \\
\hline & $40: 2$ & 842.5917 & 42.531 & 1.04939 & 0.006 \\
\hline & O-38:6 & 792.5185 & 42.863 & 1.47594 & 0.011 \\
\hline LPS & $18: 0$ & 524.2994 & 44.735 & 1.72409 & 0.006 \\
\hline \multirow[t]{12}{*}{ SM } & $16: 1$ & 745.5501 & 49.997 & 1.02742 & 0.008 \\
\hline & $16: 0$ & 747.5657 & 49.915 & 1.00037 & 0.014 \\
\hline & $16: 0$ & 749.5814 & 49.808 & 1.15175 & 0.002 \\
\hline & 18:0 & 777.6127 & 49.523 & 1.37744 & 0.001 \\
\hline & $20: 0$ & 803.6283 & 49.547 & 1.0511 & 0.007 \\
\hline & $22: 1$ & 829.6440 & 49.381 & 1.12875 & 0.002 \\
\hline & $22: 0$ & 831.6596 & 49.215 & 1.10256 & 0.011 \\
\hline & $23: 1$ & 843.6595 & 49.310 & 1.03336 & 0.012 \\
\hline & $24: 2$ & 855.6595 & 49.298 & 1.06408 & 0.010 \\
\hline & $24: 1$ & 857.6752 & 49.191 & 1.07553 & 0.008 \\
\hline & $24: 0$ & 859.6909 & 49.108 & 1.1364 & 0.008 \\
\hline & $26: 2$ & 883.6908 & 49.014 & 1.14231 & 0 \\
\hline \multirow[t]{5}{*}{ PI } & $34: 1$ & 835.5342 & 42.815 & 1.00607 & 0.035 \\
\hline & $36: 0$ & 865.5812 & 42.720 & 1.05153 & 0.006 \\
\hline & $38: 5$ & 883.5342 & 42.910 & 1.16534 & 0 \\
\hline & $38: 3$ & 887.5655 & 42.661 & 1.04875 & 0.026 \\
\hline & $38: 2$ & 889.5812 & 42.329 & 1.29563 & 0.020 \\
\hline
\end{tabular}

HPLC-ESI-QTOF-MS was used to detect the phospholipid components of cells. Mass Hunter Data Acquisition b.02.00 was used to collect and analyze the Data. Partial least-squares discriminant Analysis (PLS-DA) was performed on simca-13.0 to screen phospholipid components with VIP value greater than 1 .

Table 2. Comparison of the phospholipids in the different NTE expression cells. SH/dsRNA-NTE vs SK-N-SH SH/HA-NEST vs SK-N-SH

\begin{tabular}{ccccc}
\hline $\begin{array}{c}\text { Phospholipids } \\
\text { category }\end{array}$ & Fold change & $P$ value & Fold change & $P$ value \\
\hline PC & 1.40 & 0.009 & 0.67 & 0.006 \\
LPC & 0.93 & 0.621 & 0.58 & 0.058 \\
PI & 1.35 & 0.037 & 0.61 & 0.036 \\
PG & 0.90 & 0.778 & 1.22 & 0.498 \\
LPG & 1.00 & 0.759 & 1.04 & 0.043 \\
PS & 1.29 & 0.025 & 0.69 & 0.247 \\
LPS & 0.80 & 0.508 & 1.57 & 0.034 \\
PE & 0.74 & 0.038 & 1.27 & 0.262 \\
LPE & 0.90 & 0.521 & 0.81 & 0.044 \\
SM & 1.42 & 0.039 & 0.66 & 0.645 \\
\hline
\end{tabular}


NTE is widely expressed in tissues and anchored in the endoplasmic reticulum of cells. NTE plays a variety of roles in the development of neurite growth and vascular formation [8]. In the adult, NTE is involved in neuronal axon maintenance [15] and the regulation of permeability in the kidney [16]. Moreover, conditional knockout of NTE in the mouse CNS can produce empty blisters and other degenerative changes in large neurons in the hippocampus, thalamus, and cerebellum [17]. In humans, NTE mutations cause a variety of neurodegenerative diseases, including spastic paraplegia and blindness [18]. In this study, NTE in the SK-N-SH cells was successfully changed. Our phospholipid omics analysis showed that the NTE changes can disturb cellular phospholipid content. As expected, the PC level was significantly elevated when NTE inhibited. PC is an important component of cell membranes and lung surfactants, and it is more common on the outer lobes of the phospholipid bilayer. PC transferase is a carrier that transports PC from membrane to membrane. PC also plays an important role in cell signaling pathways. PC is a vital biological molecule that can be found in every cell of the human body. The disruption of PC homeostasis has been found to cause many neurodegenerative diseases [19].

As the total amount of phospholipids were not affected by the changes in NTE, an increase in PC should be accompanied by a decrease in other phospholipids (Figure 4). Within the biological existence of phospholipids, the content of PE, which is secondary only to PC, decreased significantly. However, SM synthesized from PC and ceramide significantly increased [20]. PS showed a similar change with SM. These results indicate that NTE affected cell phospholipid homeostasis by interfering with phospholipid biosynthesis and mutual transformation. The present results were consistent with the findings in chicken [11]. The regulation of phospholipid homeostasis is very complicated and its specific mechanism needs to be further studied.

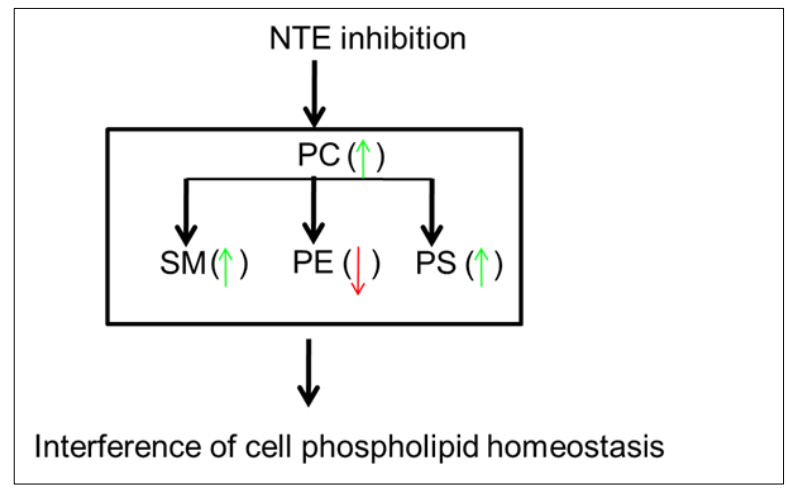

Figure 4. Schematic diagram of the hypothesis that changes in NTE affected phospholipids homeostasis. The green arrow indicated an increase in phospholipids content and the red arrow indicated a decrease in phospholipids content

\section{Conclusions}

In sum, we uncovered that cell phospholipid homeostasis was disturbed by the changes in NTE. The cell phospholipid change was similar to the changes in OPIDN sensitive animals, like chicken. Therefore, we can use the SK-N-SH cell model to explore the mechanism of changes in the phospholipid homeostasis in the initiation of OPIDN, which is much easier than the vivo experiments in practice.

Acknowledgements.All authors have read and agreed to review the manuscript. The work was funded by the National Natural Science Foundation of China (No. 31472007, 31970416).

\section{References}

1. JOHNSON, M. K., The delayed neurotoxic effect of some organophosphorus compounds. Identification of the phosphorylation site as an esterase. Biochem. J., 114(4), 1969, 711-717.

2. RICHARDSON, R. J., HEIN, N. D., WIJEYESAKERE, S. J., FINK, J. K., MAKHAEVA, G. F., Neuropathy target esterase (NTE): overview and future. Chem. Biol. Interact., 203(1), 2013, 238-244.

3. MÜHLIG-VERSEN, M., DA CRUZ, A. B., TSCHÄPE, J. A., MOSER, M., BÜTTNER, R., 
ATHENSTAEDT, K., GLYNN, P., KRETZSCHMAR, D., Loss of Swiss cheese/neuropathy target esterase activity causes disruption of phosphatidylcholine homeostasis and neuronal and glial death in adult Drosophila. J. Neurosci., 25(11), 2005, 2865-2873.

4. GLYNN, P., Neuronal phospholipid deacylation is essential for axonal and synaptic integrity. Biochim. Biophys. Acta, 1831(3), 2013, 633-641.

5. GLYNN, P., Axonal degeneration and neuropathy target esterase. Arh. Hig. Rada. Toksikol., 58(3), 2007, 355-358.

6. CHANG, P. A., WU, Y. J., Neuropathy target esterase: an essential enzyme for neural development and axonal maintenance. Int. J. Biochem. Cell Biol., 42(5), 2010, 573-575.

7. ZACCHEO, O., DINSDALE, D., MEACOCK, P. A., GLYNN, P., Neuropathy target esterase and its yeast homologue degrade phosphatidylcholine to glycerophosphocholine in living cells. J. Biol. Chem., 279(23), 2004, 24024-24033.

8. MOSER, M., LI, Y., VAUPEL, K., KRETZSCHMAR, D., KLUGE, R., GLYNN, P., BUETTNER, R., Placental failure and impaired vasculogenesis result in embryonic lethality for neuropathy target esterase-deficient mice. Mol. Cell. Biol., 24(4), 2004, 1667-1679.

9. CHANG, P. A., CHEN, Y. Y., QIN, W. Z., LONG, D. X., WU, Y. J., The role of cell cycle-dependent neuropathy target esterase in cell proliferation. Mol. Biol. Rep., 38(1), 2011, 123-130.

10. VAN TIENHOVEN, M., ATKINS, J., LI, Y., GLYNN, P., Human neuropathy target esterase catalyzes hydrolysis of membrane lipids. J. Biol. Chem., 277(23), 2002, 20942-20948.

11. ZHU, L., WANG, P., SUN, Y. J., XU, M. Y., WU, Y. J., Disturbed phospholipid homeostasis in endoplasmic reticulum initiates tri-o-cresyl phosphate-induced delayed neurotoxicity. Sci. Rep., 6, 2016, 37574.

12. JOHNSON, M. K., Improved assay of neurotoxic esterase for screening organophosphates for delayed neurotoxicity potential. Arch. Toxicol., 37(2), 1977, 113-115.

13. BLIGH, E. G., DYER, W. J., A rapid method of total lipid extraction and purification. Can. J. Biochem. Physiol., 37(8), 1959, 911-917.

14. NIE, H., LIU, R., YANG, Y., BAI, Y., GUAN, Y., QIAN, D., WANG, T., LIU, H., Lipid profiling of rat peritoneal surface layers by online normal- and reversed-phase 2D LC QToF-MS. J. Lipid Res., 51(9), 2010, 2833-2844.

15. READ, D. J., LI, Y., CHAO, M. V., CAVANAGH, J. B., GLYNN, P., Neuropathy target esterase is required for adult vertebrate axon maintenance. J. Neurosci., 29(37), 2009, 11594-11600.

16. GALLAZZINI, M., FERRARIS, J. D., KUNIN, M., MORRIS, R. G., BURG, M. B., Neuropathy target esterase catalyzes osmoprotective renal synthesis of glycerophosphocholine in response to high NaCl. Proc. Natl. Acad. Sci. USA, 103(41), 2006, 15260-15265.

17. AKASSOGLOU, K., MALESTER, B., XU, J., TESSAROLLO, L., ROSENBLUTH, J., CHAO, M. V., Brain-specific deletion of neuropathy target esterase/swisscheese results in neurodegeneration. Proc. Natl. Acad. Sci. USA, 101(14), 2004, 5075-5080.

18.SYNOFZIK, M., KERNSTOCK, C., HAACK, T. B., SCHÖLS, L., Ataxia meets chorioretinal dystrophy and hypogonadism: Boucher-Neuhäuser syndrome due to PNPLA6 mutations. J. Neurol. Neurosurg Psychiatry, 86(5), 2015, 580-581.

19. MORGAN, N. V., WESTAWAY, S. K., MORTON, J. E., GREGORY, A., GISSEN, P., SONEK, S., CANGUL, H., CORYELL, J., CANHAM, N., NARDOCCI, N., ZORZI, G., PASHA, S., RODRIGUEZ, D., DESGUERRE, I., MUBAIDIN, A., BERTINI, E., TREMBATH, R. C., SIMONATI, A., SCHANEN, C., JOHNSON, C. A., LEVINSON, B., WOODS, C. G., WILMOT, B., KRAMER, P., GITSCHIER, J., MAHER, E. R., HAYFLICK, S. J., PLA2G6, encoding a phospholipase A2, is mutated in neurodegenerative disorders with high brain iron. Nat. Genet., 38(7), 2006, 752-754.

20. CHAKRABORTY, M., JIANG, X. C., Sphingomyelin and its role in cellular signaling. Adv. Exp. Med. Biol., 991, 2013, 1-14.

Manuscript received: 10.07 .2020 
